The Nepali Math. Sc. Report

Vol. 35, No.1 and 2, 2018

\title{
ABSTRACT EARLIEST ARRIVAL TRANSSHIPMENT WITH NETWORK RECONFIGURATION
}

\author{
RAM CHANDRA DHUNGANA ${ }^{1}$ AND TANKA NATH DHAMALA ${ }^{2}$ \\ ${ }^{1,2}$ Central Department of Mathematics, Tribhuvan University, Kathmandu, Nepal \\ ${ }^{1}$ dhungana.ramchandra@gmail.com and ${ }^{2}$ amb.dhamala@daadindia.org
}

\begin{abstract}
The abstract flow model is the generalization of network flow model which deals with the flow paths (routes) satisfying the switching property. Contraflow model increases the flow value by reversing the required arc directions from the sources to the sinks. In this paper, we integrate the concepts of abstract flow and contraflow to introduce abstract earliest arrival transshipment contraflow model in multi-terminal abstract network. The abstract contraflow on multi-terminal dynamic network is NP-Complete. We present an efficient approximation algorithm to solve the problem. This approach satisfies the demand of sinks by sending optimal flow at every possible time point and seeks to eliminate the crossing conflicts.
\end{abstract}

Key Words: Abstract flow, contraflow, earliest arrival flow, transshipment, evacuation network MSC(2010) Primary: 90B10, 90C27, 68Q25; Secondary: 90B06, 90B20.

\section{INTRODUCTION}

The first network flow model was developed in [6]. After the development of maximal static flow and maximum dynamic flow models, Different researchers have studied several network flow problems for evacuation planning. The maximum dynamic flow problem to shift maximal amount in a given time, the earliest arrival flow problem to maximize the number of evacuees in every possible time, the quickest flow problem for allocating the evacuees to a safer zone in minimum time, the lexicographically maximum dynamic flow problem to send maximum number of evacuees in given priority order within the given time period, are few examples. According to Hoffman [7], the abstract flow generalizes the concept of paths by replacing the underlying network configuration. This tactic makes the use of switching property that eliminates the crossing at intersections. The maximum weighted abstract flow model has been developed by Hoffman [7] and solved by Martens and McCormick [12]. Kappmeier et al. [10] have investigated the maximum dynamic abstract flow problem and its solution procedure. The existence of the lexicographically maximum abstract flow problem for prioritized terminals has been shown in Kappmeier [9]. The earliest arrival abstract flow introduced and solved in [9] maximize the dynamic abstract flow from the source to the sink at every possible time. The abstract earliest arrival transshipment has been approximated for the fixed demands and supplies in [9]. Zhao et al. [23] deliberate the important lane-based routing strategy for reducing the interruptions that reduce (or eliminate) crossing and merging conflicts at nodes. Selecting and turning options at nodes are limited to expand traffic flow away from a unsafe area in a lane-based routing plan.

The contraflow approach introduced in [11] is another emerging and widely accepted model for evacuation planning. It increases the outbound road capacities by reversing the direction of roads towards the safe destinations. They give programming formulation and presented a greedy heuristic and a bottleneck relief heuristic for large scale evacuations that find high quality solution. They proved that problem of reducing

Received July 17, 2018 
the egress time on evacuation network is $\mathcal{N} \mathcal{P}$-hard. The first heuristic solves a minimum cost problem in the time-expanded network in the given time period to record the total number of flow units that pass through each arc during the evacuation time and flips the direction of each arc in favour of the direction of larger flows. The use of contraflow approach is not only bounded in evacuation planning, it also can be used in traffic arrangements and to reduce road accidents. Several mathematical models, heuristics, optimization and simulation techniques taking into account of macroscopic and microscopic behavioural characteristics deal with contraflow for this transportation network, however a suitable contraflow solution is still lacking due to very high computational costs.

The maximum dynamic contraflow problem has been introduced in [20] and solved with polynomial time algorithm for two terminal general network. The maximum dynamic contraflow problem on multi-terminal network is $\mathcal{N} \mathcal{P}$-complete, [20]. Authors in [3, 17, 16] presented the earliest arrival, quickest transshipment and lex-maximum dynamic contraflow problems in both discrete and continuous time settings. The earliest arrival contraflow on series-parallel network can be solved in strongly polynomial time but the procedures on general networks is pseudo-polynomial. The quickest transshipment contraflow problem has been solved in polynomial time. For the given priority ordering, the lex-maximum dynamic contraflow problem is solved in polynomial time for multi-terminal network. For the fixed supplies and demands, the earliest arrival transshipment contraflow has been introduced in $[18,16]$ in discrete and continuous times. They solved the problems in multi-source and single-sink network as well as single-source and multi-sink network. In both cases, their algorithms have polynomial time complexity. Moreover, they presented approximation algorithms to compute the approximate earliest arrival transshipment contraflow for multi-terminal networks, [18, 19]. Moreover, authors in [19] introduced the abstract contraflow approach with path reversal capability. They presented a polynomial time algorithm to solve the abstract maximum dynamic contraflow in continuous time setting. Authors in [15] investigated quickest contraflow problems with constant and load dependent transit times. Various network flow models have been extended to the partial contraflow models in [14]. They have also presented solution procedures for different partial contraflow problems. For more details, we refer to $[2]$.

The lane based contraflow and crossing elimination strategies at intersections is solved by the authors [22]. As introduced in [23], a network optimization model is to integrate these problems. The study [21], formulates the bi-level lane-based network optimization and simulation model, where the upper level optimizes the network evacuation performance subject to the contraflow and crossing-elimination constraints, and the lower level simulates dynamic evacuation flows. The multi-model integrated contraflow contains non-contraflow to shorten the strategy setup time, full-lane contraflow to minimize the evacuation network capacity and bus contraflow to realize the transit cycle operation. A more realistic contraflow problem with evacuation priorities and the setup time has been considered, (see in [2]).

The notations and prior works in network flows are presented in Section 2. The abstract flow and abstract contraflow models with their solution algorithms have been discussed in Section 3. The earliest arrival abstract contraflow model on multi-terminal abstract network has been introduced in Section 4. Also, the 2- value approximation algorithm for the problem is proposed. Section 5 concludes the paper.

\section{Denotations and Basic Models}

2.1. Abstract Network Flows. Consider a multi-terminal evacuation network $N=(E, \Gamma, b, \tau, S, D, T)$ where $E$ and $\Gamma$ represent the sets of elements and paths, respectively. Let $b: E \longrightarrow Z^{+}$be the capacity function and $\tau: E \longrightarrow Z^{+}$be the transit time function. The given non-negative time horizon $T$ is symbolized by $T=\{0,1, \cdots, T\}$ in discrete time setting, whereas it is denoted by $T=\{[0,1), \cdots,[T, T+1)\}$ in continuous time setting. For every path $\gamma \in \Gamma$ there is a linear order $<_{\gamma}$ of elements and the set of such paths $\Gamma$ satisfies the switching property in abstract network setting. A switching property requires that for each $\gamma_{1}, \gamma_{2} \in \Gamma$ and $e \in \gamma_{1} \cap \gamma_{2}$, there exist paths $\gamma_{1} \times_{e} \gamma_{2} \subseteq\left\{a \in \gamma_{1}: a \leqslant \gamma_{1} e\right\} \cup\left\{a \in \gamma_{2}: a \geqslant \gamma_{2}\right.$ $e\}$ and $\gamma_{2} \times_{e} \gamma_{1} \subseteq\left\{a \in \gamma_{2}: a \leqslant \gamma_{2} e\right\} \cup\left\{a \in \gamma_{1}: a \geqslant_{\gamma_{1}} e\right\}$. The before and after parts from $e$ of the path $\gamma$ excluding $e$ are denoted by $(\gamma, e)=\left\{p \in \gamma: p<_{\gamma} e\right\}$ and $(e, \gamma)=\left\{p \in \gamma: p>_{\gamma} e\right\}$, respectively. Let 
$f: \Gamma \longrightarrow R^{+}$be the flow function. The generalized weighted maximum abstract flow problem formulated in [7] optimizes the value of Equation 2.1 satisfying Constraints 2.2 and 2.3.

$$
\begin{aligned}
\max \sum_{\gamma \in \Gamma} \omega(\gamma) f(\gamma) & \\
\sum_{\gamma \in \Gamma: e \in \gamma} f(\gamma) & \leq b_{e}, \forall e \in E \\
f(\gamma) & \geq 0, \forall \gamma \in \Gamma
\end{aligned}
$$

The weight function $\omega: \Gamma \longrightarrow R^{+}$generalize the maximum abstract flow problem by specifying the reward per unit of flow sent along each path. The choice of the weighted function $\omega$ is restricted to supermodular functions, i.e., $\omega\left(\gamma_{1} \times_{e} \gamma_{2}\right)+\omega\left(\gamma_{2} \times_{e} \gamma_{1}\right) \geq \omega\left(\gamma_{1}\right)+\omega\left(\gamma_{2}\right)$ for every $\gamma_{1}, \gamma_{2} \in \Gamma$ and $e \in \gamma_{1} \cap \gamma_{2}$, as the general problem is $\mathcal{N} \mathcal{P}$-hard. The generalized minimum weight abstract cut is the dual of the generalized maximum weighted abstract flow problem, [7], which can be formulated as:

$$
\begin{aligned}
\min \sum_{e \in E} b_{e} y(e) & \\
\sum_{e \in \gamma} y(e) & \geq \omega(\gamma), \forall \gamma \in \Gamma, \\
y(e) & \geq 0, \forall e \in E
\end{aligned}
$$

where a value $y(e)$ is assigned to every element $e \in E$ covering every path according to its weight.

The consideration of time factor transforms the abstract flow model into dynamic abstract flow model. The dynamic abstract flow problems can be transformed into static network by constructing the corresponding time expended networks. The time expanded network can violate the switching property. So, Kappmeier et al. [10] introduced the holdover of flow at intermediate nodes to construct an abstract time expanded network. Suppose $\sigma: \Gamma \rightarrow\{1,2, \cdots, T\}$ be waiting times for every elements of $\Gamma$. Then the flow enters to $e \in \gamma$ at time $\sum_{q \in \gamma \rightarrow e}\left(\sigma_{q}+\tau_{q}\right)+\tau_{e}$ as it travelling along $\gamma$ waits $\sigma_{q}$ time units before passing through $e$. The set $\gamma^{\sigma}=\left\{e^{\kappa} \in E^{T} \mid e \in \gamma, \sum_{q \in \gamma \rightarrow e}\left(\sigma_{q}+\tau_{q}\right)+\tau_{e}=\kappa\right\}$ represents temporal paths with intermediate waiting and satisfy $q^{\kappa}<_{\gamma^{\sigma}} e^{\kappa^{\prime}}$ if and only if $q<_{\gamma} e$. Such paths arrived within $T$ is denoted by $\Gamma_{T}^{\sigma}=\left\{\gamma^{\sigma} \mid \gamma \in \Gamma, \sigma \in\{1,2, \cdots, T\}^{\Gamma}, \sum_{e \in \gamma}\left\{\sigma_{e}+\tau_{e}\right\} \leq T\right\}$. The set $\left(E_{T}, \Gamma_{T}^{\sigma}\right)$ represents the abstract time expanded network of $(E, \Gamma)$.

Suppose $f_{d y n}: \Gamma_{T}^{\sigma} \rightarrow R_{+}$be the dynamic abstract flow. The feasible dynamic abstract flow satisfies the capacity of every element at every point of time. The maximum dynamic abstract flow problem maximizes the total flow value respecting the given restrictions, [10].

$$
\begin{aligned}
\max \sum_{\gamma_{t} \in \Gamma_{T}^{\sigma}} f_{d y n}\left(\gamma_{t}\right) & \\
\sum_{\gamma_{t} \in \Gamma_{T}^{\sigma}:(e, \theta) \in \gamma_{t}} f_{d y n}\left(\gamma_{t}\right) & \leq b_{e}, \forall e \in E, \theta \in \mathbf{T} \\
f_{\text {dyn }}\left(\gamma_{t}\right) & \geq 0, \forall \gamma_{t} \in \Gamma_{T}^{\sigma}
\end{aligned}
$$

The dynamic cut is $C_{d y n}=\left\{(e, \theta) \in E_{T}: \alpha(e) \leq \theta<\alpha(e)+\tilde{y}(e)\right\}$, where $\tilde{y}$ is static weighted abstract dual integral optimal solution with weight $\omega(\gamma)$ and $\alpha(e):=\min _{\gamma \in \Gamma} \sum_{\gamma \in(\Gamma, e)}(\tau(\gamma)+\tilde{y}(\gamma))$.

Suppose $\gamma_{1}, \gamma_{2} \in \Gamma$ are two paths with a common element $e \in \gamma_{1} \cap \gamma_{2}$. The abstract network is terminal respecting, if all paths $\gamma \in \gamma_{1 \rightarrow e} \cup \gamma_{2_{e} \rightarrow}$ satisfy first $(\gamma)=$ first $\left(\gamma_{1}\right)$ and last $(\gamma)=\operatorname{last}\left(\gamma_{2}\right)$. Let $d^{+}: E \rightarrow Q_{+}$ be supplies for source elements and let $d^{-}: E \rightarrow Q_{+}$be demands for sink elements. An abstract flow satisfies given supplies and demands if $\sum_{\gamma \in \Gamma: e=f \operatorname{first}(\gamma)} f_{\gamma}=d_{e}^{+}$and $\sum_{\gamma \in \Gamma: e=\operatorname{last}(\gamma)} f_{\gamma}=d_{e}^{-}$holds for source and sink elements, respectively. It is possible that an element is a source and a sink both.

Let $f$ be an abstract flow in $\left(E_{T}, \Gamma_{T}^{\sigma}\right)$. For any $t \in\{1,2, \cdots, T\}$, consider the set of temporal paths $\Gamma_{t}^{\sigma}=\left\{\gamma \in \Gamma \mid \sum_{e \in \gamma}\left\{\sigma_{e}+\tau_{e}\right\} \leq t\right\}$. Define the flow with value $\left|f_{d y n}\right|_{t}=\sum_{\gamma \in \Gamma^{t}} f_{\gamma}$ that arrives until time $t$. An earliest arrival abstract flow problem is to find a maximum flow such that it holds $\left|f_{d y n}\right|_{t} \geq\left|f_{d y n}^{t}\right|$ for all $t$, where $\left|f_{d y n}^{t}\right|$ is the value of a maximum dynamic abstract flow with time horizon $t$. 
2.2. Contraflow Reconfiguration. The core concept behind the contraflow technique is to improve the outbound capacity by adopting the path reversals toward the sinks keeping the same travel time in the network. As a result the flow value is increased and egress time is degreased. Let $\Gamma=\{\vec{\gamma}, \overleftarrow{\gamma}\}$ be the set of all paths in contraflow abstract network $N$ with capacities $b(\vec{\gamma})=\min \left\{b_{e}: e \in \vec{\gamma}\right\}$ and $b(\overleftarrow{\gamma})=\min \left\{b_{e}: e \in\right.$ $\overleftarrow{\gamma}$ \}. We define the undirected auxiliary network $\tilde{N}$ by adding the capacities on the corresponding two-way paths and keeping the transit time (if any) fixed. The set of elements and paths are denoted by $\tilde{E}$ and $\tilde{\Gamma}$, where $\tilde{e} \in \tilde{E}$ and $\tilde{\gamma} \in \tilde{\Gamma}$. Then the capacity function is defined as $b(\tilde{\gamma})=\min \left\{b_{\tilde{e}}: \tilde{e} \in \tilde{\gamma}\right\}$ while the travel times (if any) on paths remains the same. The auxiliary network resulting from path reversal holds order of elements for each $\tilde{\gamma} \in \tilde{\Gamma}$ and $\tilde{\Gamma}$ satisfies the switching property. Thus, the auxiliary network of the abstract evacuation network is also an abstract network.

\section{Abstract Contraflow Problems}

In this section, we discuss abstract flow, contraflow and abstract contraflow problems with their solution status from different literatures. The contraflow approach makes use of path reversals in abstract network at time zero without any switching costs.

Example 3.1. Let $E=\{s, a, b, z\}$ and $\Gamma=\left\{\overrightarrow{\gamma_{1}}, \overrightarrow{\gamma_{2}}, \overrightarrow{\gamma_{3}}, \overrightarrow{\gamma_{4}}, \overleftarrow{\gamma_{1}}, \overleftarrow{\gamma_{2}}, \overleftarrow{\gamma_{3}}, \overleftarrow{\gamma_{4}}\right\}$ with $\overrightarrow{\gamma_{1}}=\{s, a, z\} ; \overrightarrow{\gamma_{2}}=\{s, a, b, z\}$ $\overrightarrow{\gamma_{3}}=\{s, b, z\} ; \overrightarrow{\gamma_{4}}=\{s, b, a, z\} ; \overleftarrow{\gamma_{1}}=\{z, a, s\} ; \overleftarrow{\gamma_{2}}=\{z, b, a, d\} ; \overleftarrow{\gamma_{3}}=\{z, b, s\}$ and $\overleftarrow{\gamma_{4}}=\{z, a, b, s\}$ and capacities $4,2,2,2,3,3,4$ and 2 , respectively. We forget the direction of paths and reformulate it by adding the capacities of paths between the terminals. Set of paths in abstract auxiliary network is $\tilde{\Gamma}=\left\{\tilde{\gamma_{1}}, \tilde{\gamma_{2}}, \tilde{\gamma_{3}}, \tilde{\gamma_{4}}\right\}$ (cf. Figure 1). After contraflow reconfiguration, the path set becomes $\Gamma_{R}=\left\{\overrightarrow{\gamma_{1}}, \overrightarrow{\gamma_{2}}, \overrightarrow{\gamma_{3}}, \overrightarrow{\gamma_{4}}\right\}$ with improved capacities $7,2,6$ and 3 for $\overrightarrow{\gamma_{1}}, \overrightarrow{\gamma_{2}}, \overrightarrow{\gamma_{3}}$, and $\overrightarrow{\gamma_{4}}$, respectively. Set of paths in abstract auxiliary network is $\tilde{\Gamma}=\left\{\tilde{\gamma_{1}}, \tilde{\gamma_{2}}, \tilde{\gamma_{3}}, \tilde{\gamma_{4}}, \tilde{\gamma_{5}}, \tilde{\gamma_{6}}\right\}$ (cf. Figure 1).
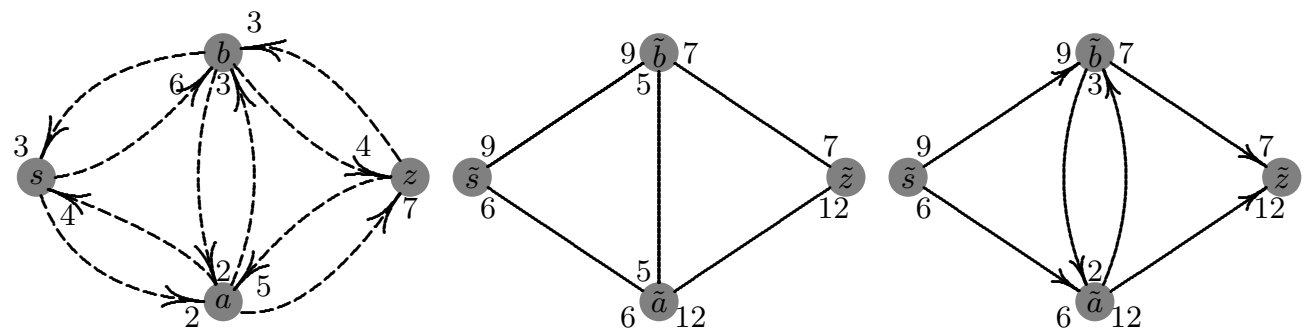

FiguRE 1. Abstract evacuation, auxiliary and reconfigured networks, respectively.

The maximum abstract flow problem is introduced in [7] and an efficient algorithm for the problem has been presented in [13]. Based on the same algorithm, the maximum abstract contraflow algorithm has been developed in [19] for the path reversal maximum abstract contraflow. The maximum abstract contraflow doubles the flow value after contraflow reconfiguration if every element in a minimum abstract cut has symmetric capacity, [19]. Kappmeier et al. [10] extended the abstract flow problem into maximum dynamic abstract flow problem and provided efficient solution procedure to solve the problem. To provide the solution procedure for the problem they have transformed the dynamic network into static by constructing time expanded network. For each interval, a copy of the element set $\tilde{E}$, the element set $\tilde{E}_{T}:=\tilde{E} \times \mathbf{T}$ will be constructed. The time expanded network is constructed by $\tilde{E}_{T}=\{(\tilde{e}, \theta) \mid \tilde{e} \in \tilde{E}, \theta \in\{1,2, \cdots, T\}\}$. The time expanded network of the dynamic abstract network is shown in Figure 2 where the numbers on the network represent transit time of the elements.

The abstract path system is allowed to be asymmetric with respect to the path capacities but the transit times are symmetric. In auxiliary network only capacities of the paths change but the transit times remain the same. As the abstract cut $C_{d y n}$ contains an element of every temporally repeated paths, the capacity constraints are satisfied at each point of time. Thus for a subset $C_{d y n} \subseteq E_{T}$, the set $\gamma_{t} \cap C_{d y n}$ is 
nonempty to each $\gamma_{t} \in \Gamma_{T},[10]$. This implies that $\sum_{\gamma_{t} \in \Gamma_{T}} f_{d y n}\left(\gamma_{t}\right) \leq \sum_{(e, \theta) \in C_{d y n}} b_{e}$. The number of paths created by applying the time expansion is linear in $T$ and thus exponential in the size of input.

Example 3.2. The time expanded network of an auxiliary network can destroy the switching property. Let $(\tilde{E}, \tilde{\Gamma})$ be an auxiliary network with $\tilde{\Gamma}=\left\{\gamma^{1}, \gamma^{2}, \gamma^{3}, \gamma^{4}\right\}$ and $\tilde{E}=\{s, a, b, z\}$, where $\gamma^{1}=(s ; a ; b ; z), \gamma^{2}=$ $(s ; b ; a ; z), \gamma^{3}=(s ; a ; z), \gamma^{4}=(s ; b ; z)$ together with their reversals. The set $\tilde{\Gamma}$ satisfies the switching and order properties. For $T=4$, we have $\Gamma_{T}=\left\{\gamma_{0}^{1}, \gamma_{2}^{1}, \gamma_{0}^{2}, \gamma_{1}^{2}, \gamma_{0}^{3}, \gamma_{1}^{3}, \gamma_{2}^{3}, \gamma_{0}^{4}, \gamma_{1}^{4}, \gamma_{2}^{4}\right\}$ with $\gamma_{0}^{1}=$ $\{(s, 0),(a, 1),,(b, 2),(z, 3)\}$ and $\gamma_{1}^{2}=\{(s, 1),(b, 2),(a, 3),(z, 4)\}$. But time expansion of given network does not contain $\gamma_{0}^{1} \times_{(b, 2)} \gamma_{1}^{2}$ (cf. Figure 2).

Internal waiting should be allowed to construct abstract time expanded network. According to [10], the waiting has no influence on the optimality and the temporally repeated optimal solutions can be obtained even if waiting is allowed internally on time expanded network. Based on this idea, an efficient algorithm for the maximum dynamic abstract contraflow problem has been developed in [4]. The existence lexicographically maximum abstract flow and a polynomial time algorithm for the problem is given by Kappmeier [9]. In his model, the order of terminals has to fulfill compatible property if more than one terminal node is contained in a path. The existence of earliest arrival abstract flow described by Kappmeier [9] generalizes the earliest arrival flow and maximizes the dynamic abstract flow at each possible time point. For the lexicographically maximum abstract contraflow and earliest arrival abstract contraflow problems with their algorithms, we refer to [4].
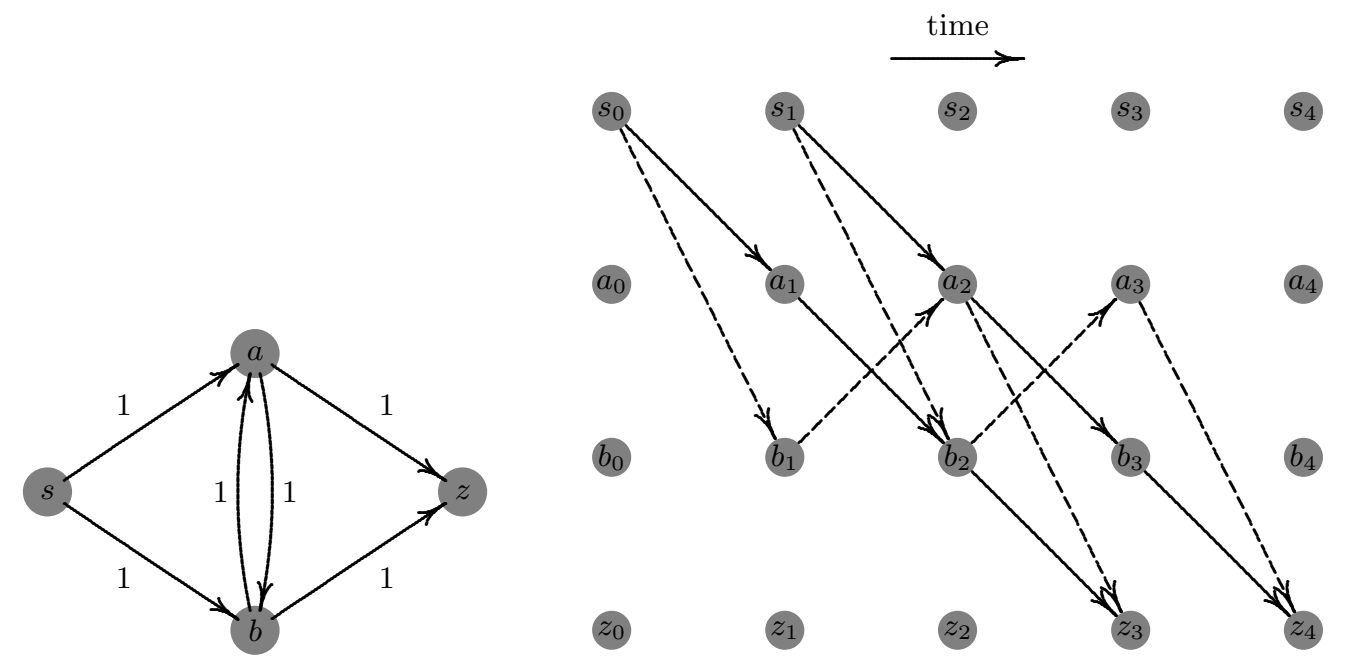

Figure 2. Dynamic abstract network and time expanded network, respectively.

\section{Abstract Earliest Arrival Transshipment Contraflow}

We study abstract earliest arrival contraflow problem on multi-terminal network. Clearly, the abstract earliest arrival contraflow cannot be obtained for multiple sinks. But the flow value can be approximated by adding supplies and demands for source and sink elements, respectively. Based on the results of $[9,17]$ on contraflow and abstract flow, we introduce abstract earliest arrival contraflow Problem 4.1 and propose an approximation Algorithm 4.3 to solve it. Recall that, the abstract contraflow transshipment having the earliest arrival property do not exist.

Problem 4.1. Let $N=(G, b, \tau, S, D, T)$ be an abstract network, where $G=(E, \Gamma)$. The multi-terminal abstract earliest arrival contraflow problem is to find an abstract earliest arrival flow with path reversals capability that satisfies the supplies and demands at terminals. 
Example 4.1. Consider an abstract network with elements $E=\left\{s, a, b, c, z_{1}, z_{2}\right\}$ and paths $\Gamma=\left\{\overrightarrow{\gamma_{1}}, \overrightarrow{\gamma_{2}}, \overleftarrow{\gamma_{1}}, \overleftarrow{\gamma_{2}}\right\}$ $\overrightarrow{\gamma_{1}}=\left\{s, a, c, z_{1}\right\}, \overrightarrow{\gamma_{2}}=\left\{s, a, b, z_{2}\right\}, \overleftarrow{\gamma_{1}}=\left\{z_{1}, c, a, s\right\}$ and $\overleftarrow{\gamma_{2}}=\left\{z_{2}, b, a, s\right\}$. Suppose $d_{s}^{+}=12, d_{z_{1}}^{-}=4, d_{z_{2}}^{-}=$ $8, d_{a}=0, d_{b}=0$, and $d_{c}=0$ be the balance vectors. Construct auxiliary network with $\tilde{\Gamma}=\left\{\tilde{\gamma}_{1}, \tilde{\gamma}_{2}\right\}$, $\tilde{\gamma_{1}}=\left\{\tilde{s}, \tilde{a}, \tilde{c}, \tilde{z_{1}}\right\}$ and $\tilde{\gamma_{2}}=\left\{\tilde{s}, \tilde{a}, \tilde{b}, \tilde{z_{2}}\right\}$ which is shown in Figure 3 .

Besides the given feasible transshipment at time 5 there is a feasible transshipment which sends 4 units of flow on path $\tilde{\gamma}_{1}$ at time 4, 4 units of flow on path $\tilde{\gamma}_{2}$ at time 5 and another 4 units of flow on path $\tilde{\gamma}_{2}$ at time 6 . Here, 4 units of flow arrive at time 4 because $\tilde{\gamma}_{1}$ has 3 transit time, another 4 units arrive at time 5 through path $\tilde{\gamma}_{2}$ and next 4 units arrive at time 6 through path $\tilde{\gamma}_{2}$. This solution sends 4 units of flows earlier, but needs more time to send the last 4 units of flows. Hence, no earliest arrival transshipment contraflow exists in the abstract network and the new solution is a 2 -value-approximate flow.
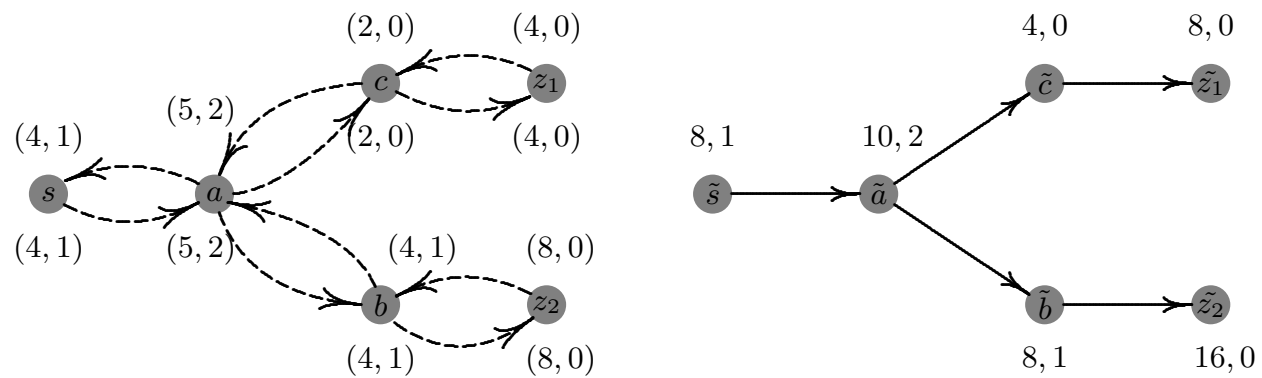

FiguRE 3. Multi-terminal abstract evacuation network and network after contraflow reconfiguration, respectively.

The proposed algorithm works on extended path system having two restrictions such that every subpaths should preserve orders and no flow can exceed the demands and supplies, [9], as constructed below. Let $\left(E_{T}, \Gamma_{T}^{\sigma}\right)$ be the abstract time expanded network for some time horizon $T$. We introduce additional super source elements $s^{*}$, super sink elements $z^{*}$ and counting elements $z_{c}$. The time-expanded ground set is defined as: $E_{T}^{*}=E_{T} \cup\left\{s_{e}^{*}: s \in S\right\} \cup\left\{z_{e}^{*}: z \in D\right\} \cup\left\{e_{c}^{\theta}: e \in D, \theta \in\{1,2, \cdots, T\}\right\}$. We extend each original temporal path $\gamma^{\sigma}=\left(e_{1}, e_{2}, \cdots, e_{n}\right) \in \Gamma_{T}^{\sigma}$ by the corresponding super terminals and a counting element. Let $s=e_{1}=\operatorname{first}(\gamma)$ and $z^{\theta}=e_{n}=\operatorname{last}(\gamma)$ be the first and last element of $\gamma_{\sigma}$, respectively. The extended path $\gamma^{* \sigma}=\left(s^{*}, e_{1}, e_{2}, \cdots, e_{n}, z_{c}^{\theta}, z^{*}\right)$ contains three more elements, the super source as new first element, the super sink as last element and the counting element left of the super sink. The set of extended paths $\Gamma_{T}^{* \sigma}$ consists of all extended paths $\gamma^{* \sigma}$ with internal waiting at intermediate elements.

Example 4.2. Figure 4 is the extended time expanded network derived from the Figure 2.

The primal dual pair $(f, y)$ satisfies $\sum_{e \gamma} y_{e}=\omega_{\gamma}-\lambda$ for each $\gamma \in \Gamma$ and $y_{e}\left(b_{e}-\sum_{e \gamma} f_{e}\right)=0$ for each $e \in E$, where $\lambda \in Q$ is a given variable. These are the relaxed optimality conditions for weighted abstract flow and weighted abstract cut, where $\lambda$ specifies deviated solutions from the optimum solution. The set of restricted elements is denoted by $R=\left\{e \in E: y_{e}>0\right\} \subseteq E$. A solution of the restricted abstract maximum flow problem is a maximum flow $f$ under the condition that the flow through restricted elements remains unchanged. The LP formulation of restricted maximum abstract flow and restricted minimum abstract cut problems presented by Kappmeier [9] are the following.

$$
\begin{aligned}
\max \sum_{\gamma \in \Gamma} f(\gamma) & \\
\sum_{\gamma \in \Gamma: e \in \gamma} f(\gamma) & \leq b_{e}, \forall e \in E \\
\sum_{\gamma \in \Gamma: e \in \gamma} f(\gamma) & =b_{e}, \forall e \in R \\
f(\gamma) & \geq 0, \forall \gamma \in \Gamma
\end{aligned}
$$




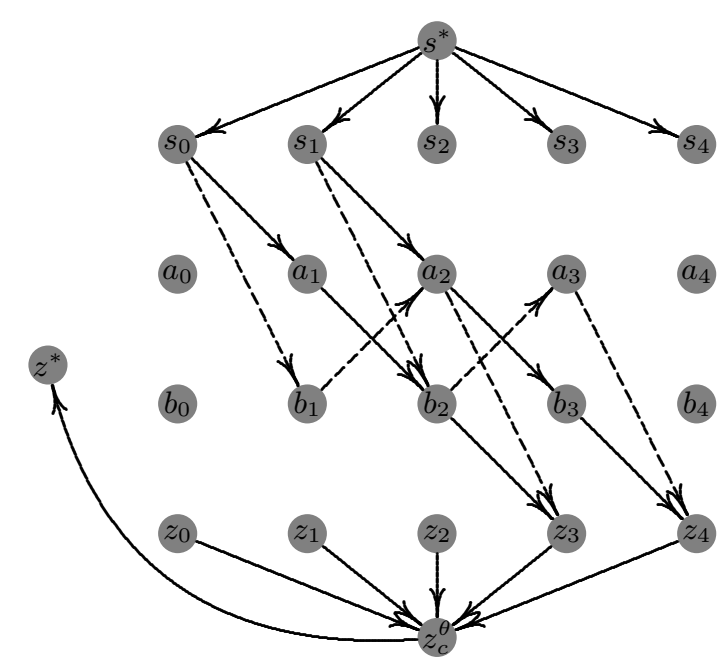

Figure 4. Extended time expanded network.

$$
\begin{aligned}
\min \sum_{e \in E} l_{e} b_{e} & \\
\sum_{e \in \gamma} l_{e} & \geq \omega(\gamma), \forall \gamma \in \Gamma \\
l_{e} & \geq 0, \forall e \in E \backslash R, \text { where } l_{e} \text { is the length of } e
\end{aligned}
$$

Let $f^{i}$ be an abstract dynamic flow in the abstract time expanded network with extended paths $\left(A^{*}{ }_{T}, \Gamma_{T}^{*^{\sigma}}\right)$ for a time horizon $i$. All paths use a counting element $z_{c}^{\theta}$ for some $\theta \in\{1,2, \cdots, i\}$. Let $v\left(z_{c}^{\theta}\right)=\sum_{\gamma: z_{c}^{\theta} \in \gamma} f_{\gamma}$ be the value of flow through the counting element. Define a new abstract time expanded network by specifying new capacities $b_{e}^{\prime}$, where $b_{e}^{\prime}=b_{e}$ for all elements $e$ that are not counting elements. The capacity of counting elements is restricted to the flow value through them, i. e., we set $b_{z_{c}^{\theta}}^{\prime}=v\left(z_{c}^{\theta}\right)$ for all counting elements $z_{c}^{\theta}$ in the time-expanded network. All paths use exactly one of the counting elements and they are all saturated. Thus, flow $f^{i}$ remains feasible in the abstract time expanded network with the new capacities $b_{e}^{\prime}$. Define a feasible dual solution by setting

$$
y_{e}=\left\{\begin{array}{ll}
b_{e}^{\prime}, & e \text { is a counting element } \\
0, & \text { otherwise }
\end{array} \text { for all } e \in E\right.
$$

The flow $f$ and dual values $y$ are feasible in the larger abstract time expanded network $\left(E_{i+1}^{*}, \Gamma_{i+1}^{* \sigma}\right)$ for a time horizon increased by 1 . The algorithm by Martens and McCormick [12] has been applied to augment flow without removing flow on the counting elements in the process of finding restricted maximum abstract flow. In the classical setting, the Triple - Optimization - Theorem, [8] states that an earliest arrival flow is equal to a minimum cost flow where the costs equal the transit times. The same idea has been applied in abstract flow model and defined rewards such that they reflect the arrival time. A path that arrives earlier has the higher reward. Paths arriving in the first time step, i. e., paths with zero travel time, have a reward of $T$. The reward decreases linearly with the arrival time and paths arriving at time $T$ have a reward of 1 . Let $\gamma^{* \sigma}$ be a path in the abstract time expanded network for time horizon $T, \theta$ be the arrival time of $\gamma^{* \sigma}$. Then the reward is defined as $w\left(\gamma^{* \sigma}\right)=T-\theta+1$ which is supermodular.

Algorithm 4.3. Approximate Abstract Earliest Arrival Contraflow Algorithm

(1) Given an abstract network $N=(G, b, \tau, T, S, D)$ with demands $d^{-}$and supplies $d^{+}$.

(2) Construct abstract auxiliary network, $\tilde{N}=\left(\tilde{G}, \tilde{b_{e}}, T, \tilde{S}, \tilde{D}\right)$ with new capacity and transit time functions $\tilde{b}(\tilde{\gamma})=b(\vec{\gamma})+b(\overleftarrow{\gamma})$ and $\tilde{\tau}(\tilde{\gamma})=\sum_{\tilde{e} \in \tilde{\gamma}} \tilde{\tau}(\tilde{e})$ 
(3) Solve the problem in the auxiliary network using the greedy abstract value-approximate earliest arrival flow algorithm, [9] as follows:

(a) Set $i=1$ and compute an abstract flow $\tilde{f}^{1}$ in $\left(\tilde{E}_{1}^{*}, \tilde{\Gamma}_{1}^{* \sigma}\right)$. Define $\lambda=1$.

(b) Let $\left(\tilde{E}_{i+1}^{*}, \tilde{\Gamma}_{i+1}^{* \sigma}\right)$ be the abstract time expanded network with extended paths for time horizon $i+1$. Define path weights $\omega\left(\tilde{\gamma}^{* \sigma}\right)=T-\theta+1$. The updated capacities and dual values are given by

$$
\begin{gathered}
b_{\tilde{e}}^{\prime}=\left\{\begin{array}{ll}
v\left(z_{c}^{\theta}\right), & \text { if } \tilde{e}=v\left(z_{c}^{\theta}\right) \text { is a counting element } \\
b_{\tilde{e}}, & \text { otherwise }
\end{array} \text { for all } \tilde{e} \in \tilde{E}_{i+1}^{*},\right. \\
y_{\tilde{e}}=\left\{\begin{array}{ll}
\omega\left(\tilde{\gamma}^{* \sigma}\right)-\lambda, & \text { if } \tilde{e} \text { is a counting element on path } \tilde{\gamma}^{* \sigma} \in\left(\tilde{E}_{i+1}^{*}, \tilde{\Gamma}_{i+1}^{* \sigma}\right) \\
0, & \text { otherwise }
\end{array} \text { for all } \tilde{e} \in \tilde{E}^{*^{i+1}} .\right.
\end{gathered}
$$

(c) Compute an abstract flow $\tilde{f}^{i+1}$ in $\left(\tilde{E}_{i+1}^{*}, \tilde{\Gamma}_{i+1}^{* \sigma}\right)$ applying the restricted abstract maximum flow minimum cut algorithm by Martens and McCormick [12].

(d) If $\tilde{f}^{i+1}$ satisfies all balances, return flow value $x^{i+1}$. Else, set $i=i+1$ and continue with $3 b$.

(4) A path $\overleftarrow{\gamma} \in \Gamma$ is reversed if and only if the flow along $\vec{\gamma} \in \Gamma$ is greater than $b(\vec{\gamma})$ or there is a non negative flow along path $\vec{\gamma} \notin \Gamma$.

Theorem 4.4. An approximate optimal solution for Problem 4.1 can be obtained by Algorithm 4.3.

Proof. The Steps 2 and 3 are feasible by definition. Step 4 is well defined; i.e. not both paths $\vec{\gamma}$ and $\overleftarrow{\gamma}$ have to be switched at a time. This is ensured by the solution of the abstract flow in auxiliary network, [9]. Switching property cancels cycle flows [19], so that there is flow along $\vec{\gamma}$ or $\overleftarrow{\gamma}$ but not in both directions at the same time. Hence, the resulting flow from Step 4 is a feasible flow with path reversals in given evacuation network $N=(G, b, \tau, T, S, D)$.

Let $\tilde{N}_{T}^{*}=\left(\tilde{E}_{T}^{*}, \tilde{\Gamma}_{T}^{* \sigma}\right)$ be the corresponding extended abstract time expanded network of $\tilde{N}=\left(\tilde{G}, \tilde{b_{e}}, T, \tilde{S}, \tilde{D}\right)$ with intermediate waiting, [10], where $T$ be a significantly large time horizon. Step 3 provides the 2-valueapproximate abstract earliest arrival flow in the extended abstract time expanded network of auxiliary abstract network which is an optimal flow for auxiliary network, [9]. The path capacity is increased by adding the capacity of both directions between the terminals and either direction of path is allowed with modified network. A 2-value approximate earliest arrival flow with path reversal in $N=(G, b, \tau, T, S, D)$ is also a feasible solution to the earliest arrival flow problem in the auxiliary network $\tilde{N}=\left(\tilde{G}, \tilde{b_{e}}, T, \tilde{T}, \tilde{D}\right)$. As the amount of flow sent from $S$ to $D$ in Step 3 is not changed in Step 4, the resulting flow is the 2-value approximate solution for Problem 4.1.

\section{Conclusion}

In this paper, we discuss abstract flow, contraflow and abstract contraflow models from various papers. Integrating abstract earliest arrival flow and contraflow, we introduced abstract earliest arrival contraflow approach with discrete time settings on multi-terminal abstract network for the first time. A 2-value approximate algorithm has been proposed for the problem assuming fixed demands and supplies on sources and sinks, respectively. Our results increase the flow values at every possible time by reducing crossing conflicts with arc reversals toward the safe destinations in evacuation planning.

Acknowledgement. The authors thank to the AvH for the Research Group Linkage Program and DAAD for partnership program. The first author would like to acknowledgement UGC, Nepal for the partial support.

\section{REFERENCES}

[1] N. Altay, W. G. Green, OR/MS Research in Disaster Operations Management, European Journal of Operational Research, 17, 475-493, 2006 
[2] T. N. Dhamala, U. Pyakurel, S. Dempe, A Critical Survey on the Network Optimization Algorithms for Evacuation Planning Problems, International Journal of Operations Research, 15(3), 101-133, 2018

[3] Dhamala T N, Pyakurel U. Earliest Arrival Contraflow Problem on Series-parallel Graphs, International Journal of Operations Research, 2013, 10(1), 1-13

[4] R. C. Dhungana, U. Pyakurel, T. N. Dhamala, Abstract Contraflow Models and Solution Procedures for Evacuation Planning, Canadian Center of Science and Education, Journal of Mathematics Research, 10(4), 89-100, 2018

[5] R. C. Dhungana, U. Pyakurel, S. R. Khadka, T. N. Dhamala, Universally Maximum Contraflow for Evacuation Planning, International Journal of Operations Research, Nepal, 4, 67-78, 2015

[6] L. R. Ford, D. R. Fulkerson, Maximal Flow Through a Network, Canadian Mathematical Society, 8, 399-404, 1956

[7] A. Hoffman, A Generalization of Max Flow-Min Cut, Mathematical Programming, 6(1), 352-359, 1974

[8] J. J. Jarvis, H. D. Ratliff, Some Equivalent Objectives for Dynamic Network Flow Problems, Management Science, 28(1), 106-109, 1982

[9] J-P. W. Kappmeier, Generalizations of Flows over Time with Applications in Evacuation Optimization. PhD Thesis, Technical University, Berlin, 2015

[10] J-P. W. Kappmeier, J. Matuschke, B. Peis, Abstract Flows over Time: A First Step Towards Solving Dynamic Packing Problems, Theoretical Computer Science, Algorithms and Computation, 544, 74-83, 2014

[11] S. Kim, S. Shekhar, M. Min, Contraflow Transportation Network Reconfiguration for Evacuation Route Planning, IEEE Transactions on Knowledge and Data Engineering, 20, 1-15, 2008

[12] M. Martens, S. T. McCormick, A Polynomial Algorithm for Weighted Abstract Flow, Integer Programming and Combinatorial Optimization, 97-111, 2008

[13] S. T. McCormick, A Polynomial Algorithm for Abstract Maximum Flow, In: Proceedings of the Seventh Annual ACM-SIAM Symposium on Discrete Algorithms, 490-497, 1996

[14] U. Pyakurel, H. N. Nath, T. N. Dhamala, Partial Contraflow with Path Reversals for Evacuation Planning, Annals of Operation Research, DOI: 10.1007/s10479-018-3031-8, 2018

[15] U. Pyakurel, H. N. Nath and T. N. Dhamala, Efficient Contraflow Algorithms for Quickest Evacuation Planning, Science China Mathematics, Doi: 10.1007/s11425-017-9264-3, 2018

[16] U. Pyakurel, T. N. Dhamala, Continuous Dynamic Contraflow Approach for Evacuation Planning, Annals of Operations Research, Springer, 253, 573-598, 2016

[17] U. Pyakurel, T. N. Dhamala, Continuous Time Dynamic Contraflow Models and Algorithms, Hindawi Publishing Corporation, Advances in Operations Research, Volume 2016, Article ID 7902460, 1-7, 2016

[18] U. Pyakurel, T. N. Dhamala, Evacuation Planning by Earliest Arrival Contraflow, Journal of Industrial and Management Optimization, 13, 487-501, 2017

[19] U. Pyakurel, T. N. Dhamala, S. Dempe, Efficient Continuous Contraflow Algorithms for Evacuation Planning Problems, Annals of Operation Research, 254, 335-364, 2017

[20] S. Rebennack, A. Arulselvan, L. Elefteriadou, P. M. Pardalos, Complexity Analysis of Maximum Flow Froblem with Arc Reversal, Journal of Combinatorial Optimization, 29, 200-216, 2010

[21] C. Xie, D. Y. Lin, S. T. Waller, A Dynamic Evacuation Network Optimization Problem with Lane Reversal and Crossing Elimination Strategies, Transportation Research Part E, 46, 295-316, 2010

[22] C. Xie, M. A. Turnquist, Lane-based Evacuation Network Optimization: An Integrated Lagrangian Relaxation and Tabu Search Approach, Transportation Research Part C, 19, 40-63, 2011

[23] X. Zhao, Z-Y. Feng, Y. Li, A. Bernard, Evacuation Network Optimization Model with Lane-based Reversal and Routing, Hindawi Publishing Corporation Advances in Operations Research Volume 2016, Article ID 1273508, 2016 